\title{
Importance of Dynamic Analysis for RCC Structures
}

\section{Gomasa Ramesh ${ }^{1} \mid$ Doddipati Srinath $^{1} \mid$ Mandala Sheshu Kumar ${ }^{1}$}

${ }^{1}$ PG Scholar, Structural Engineering, Vaagdevi College Of Engineering, Warangal, 506005, Telangana, India.

\section{To Cite this Article}

Gomasa Ramesh, Doddipati Srinath, Mandala Sheshu Kumar, "Importance of Dynamic Analysis for RCC Structures", International Journal for Modern Trends in Science and Technology, 6(8): 271-276, 2020.

Article Info

Received on 26-July-2020, Revised on 09-August-2020, Accepted on 12-August-2020, Published on 21-August-2020.

\section{ABSTRACT}

Structural dynamics is especially used in analysis of dynamic structures under different loading conditions. It is very important to know the difference between structural statics and structural dynamics. It is very important knowledge for structural engineers in the design field as well as students too, who are studying academic subject. This paper is helpful to understanding about introduction to structural dynamics. Every structure is subjected to loading. In this discussed about dynamic loads subjected to the structure and Also to know the importance of the dynamic analysis of the structure. In this also discussed about various types of degree of freedoms of dynamic structures. This paper is very helpful to gaining knowledge in structural dynamics for students, structural designers and lecturers also. This paper is also gives the general importance of structural dynamics and also give some of importance overview to the students and all.

KEYWORDS: Structural dynamic analysis, Damping, Degree of freedom, Vibrations, mass spring system.

\section{INTRODUCTION}

Structural Dynamics is the branch of Engineering. It is a very useful and important to the students, educators, designers in the field of civil engineering and structural engineering. It is a very important tool to analyzing the structures under dynamic loading conditions. Dynamic loading includes wind loads, wave loads and earthquake loads etc. With help of this we have to study and analysis the two important topics of structures. We have to study the first important one is Vibration analysis and other important one is Earthquake analysis. These two topics are very important. First all we have to know the clear difference between structural statics and dynamics. Structural statics is used for analysis the structure under static loading conditions and in this loading of the structure is constant. In structural dynamics we have to study the dynamic loading conditions of the structures and in this loading of the structure is not constant and variable with time. In this we study the vibration and types of vibration, damping, degree of freedom, and some of various types of loadings. It is helpful to structural designers to understanding about types of loads acting, designing, detailing of the structures. Structural dynamics is the effective way to design seismic analysis of the structures. Structural dynamic is the solution to dynamic structures. In this we have study boundary conditions, applied forces, external forces,support motion. So the study of structural dynamics is very important and helpful and useful to the civil and structural engineers. 


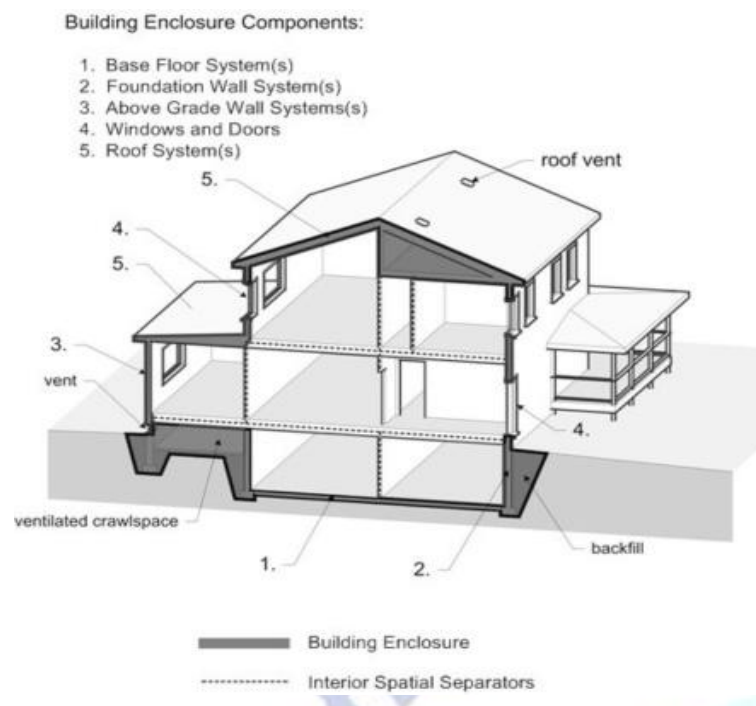

Fig. Building Components

II. LITERATURE REVIEW
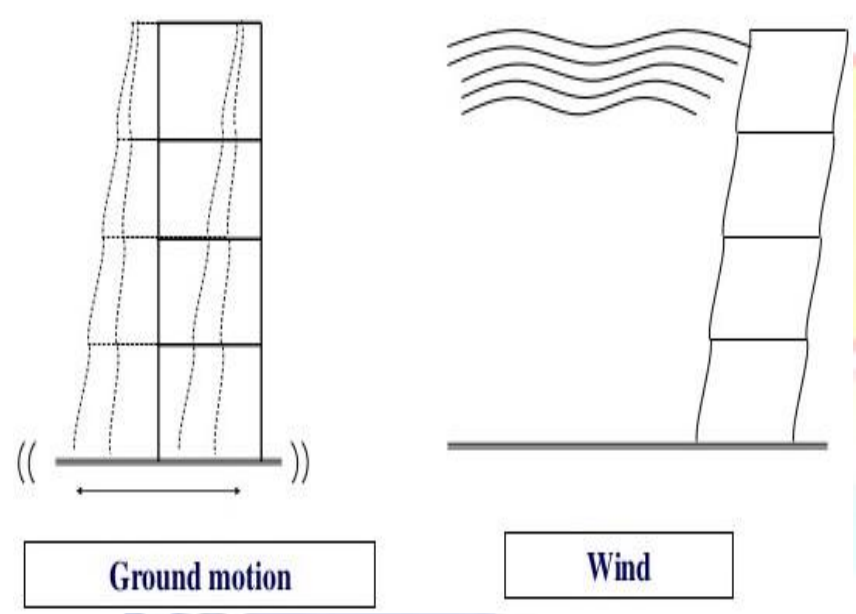

Fig. Sources of Ground Motion

\section{Fundamental Objectives of Structural Dynamic} Analysis:

1. Structures are designed safely

2. Economical

3. Design with a greater degree of freedom

4. Seismic loads are modeled accurately

5. More economical design achieved

6. Safety and serviceability

7. The Effective approach for seismic analysis of structural system

8. To know the behaviour of the structure under loading

9. Properly Analyze the structure

10. Durability and strength Aspects

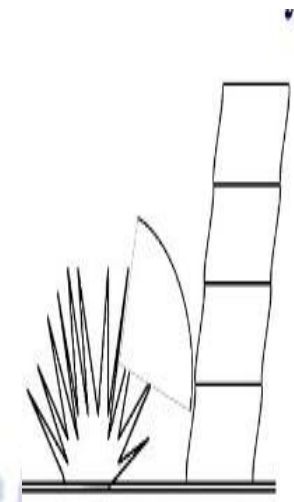

Blast?
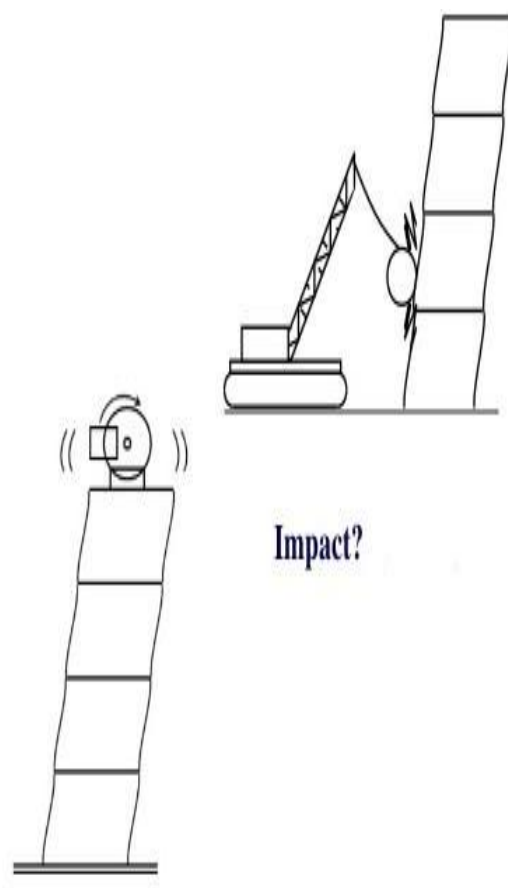

Impact?
Machine vibration

Fig. Dynamic Excitation
Basic concepts in structural dynamics:

1. Vibrations \& Oscillations

2. Free Vibration

3. Forced Vibration

4. Damping

a. With Damping

b. Without Damping

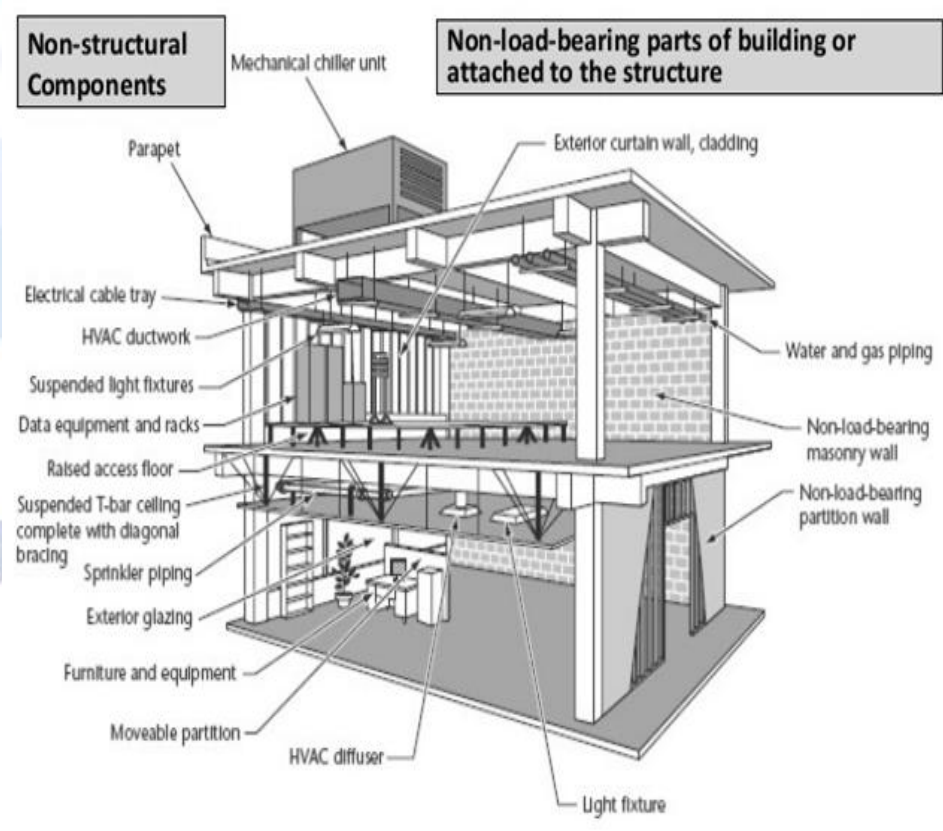

Fig. Components of RCC Structures 


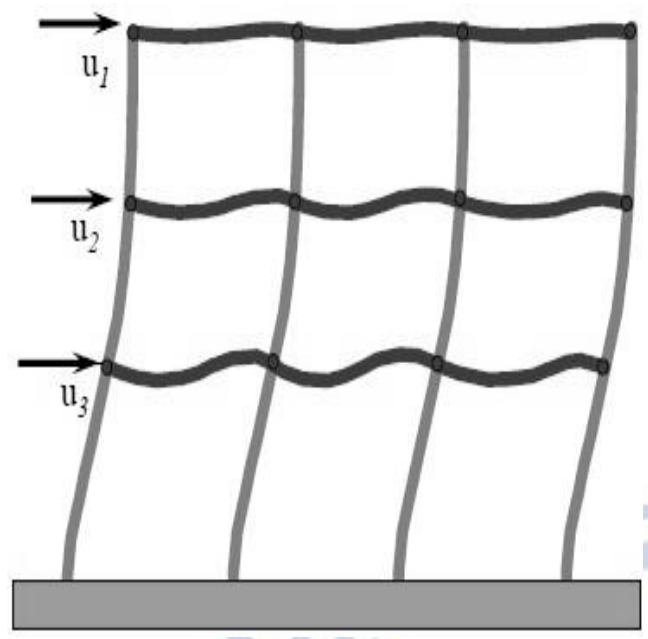

Fig. Structural Dynamics of Linear elastic system

Loading is nothing but the structure is subjected to the force. It is the application of the force to the body of the structure.

\section{Types}

1. Periodic

2. Non Periodic

3. Static

\section{Types of loadings:}

\section{Periodic loading}

The loading is does not change with respect to the time. In this variation of loading with respect to time is fully known.
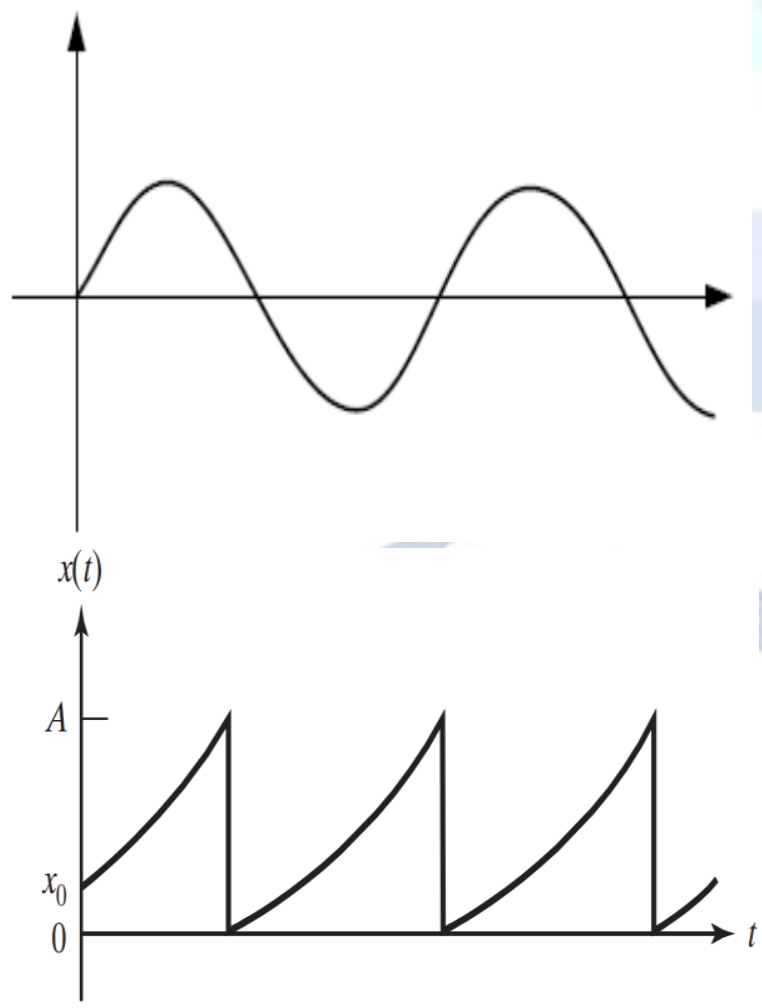

Fig. Periodic Loading

\section{Non Periodic loading}

The loading is changes with respect to the time. In this variation of loading with respect to the time is fully not known.
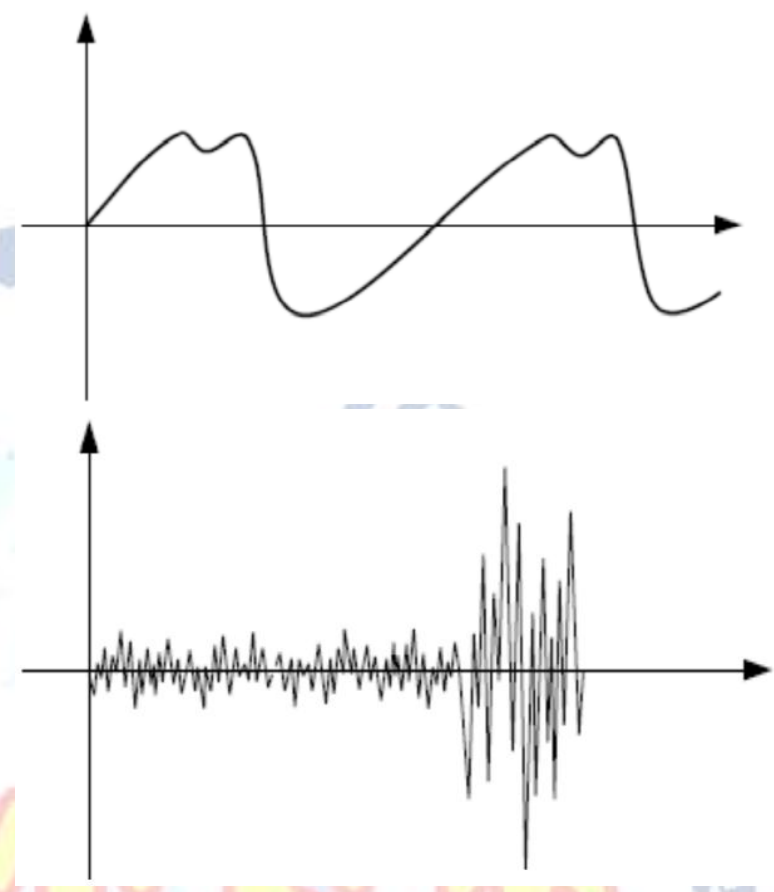

Fig. Non Priodic Loading

\section{Static Loading}

1. Static loading is the loading in which loading is not changes with respect to the time.

2. Loading is constant in nature.

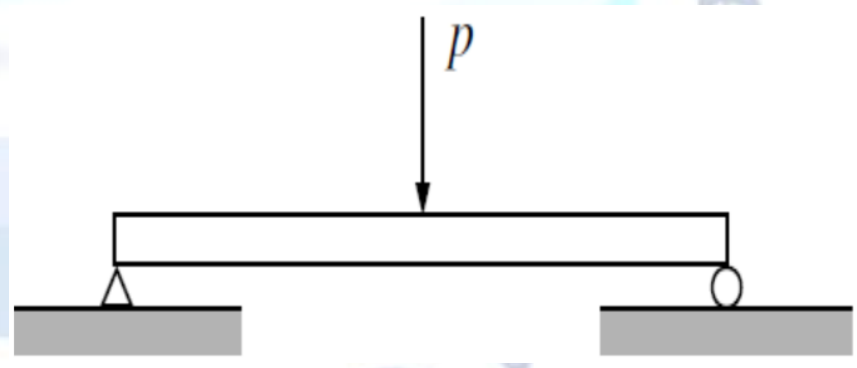

Fig. Static loading

\section{Dynamic Loading}

1. Dynamic loading is the loading in which loading is changes with respect to the time.

2. Loading is varying in nature. 


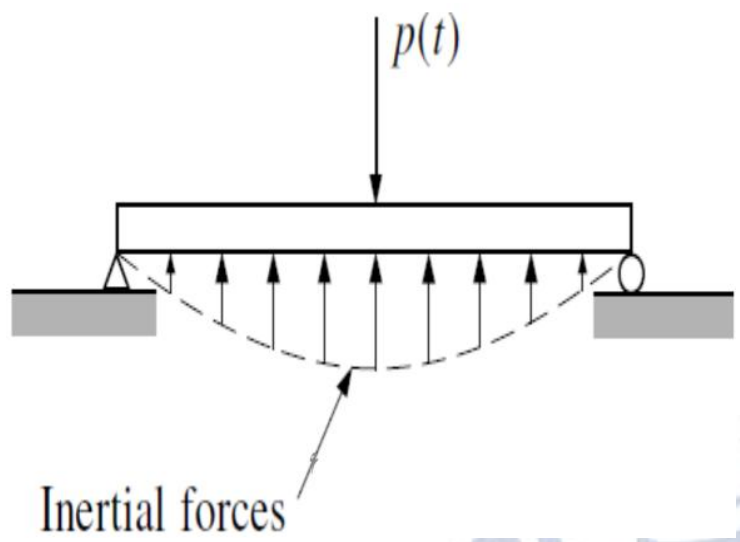

Fig. Dynamic loading

\section{METHODOLOGY}

Elements are used in the structural dynamics to know the behaviour of structural system. Elements of the Vibration system of structures are as follows;

1. Mass

2. Spring

3. Damping

4. External loads

5. Stiffness

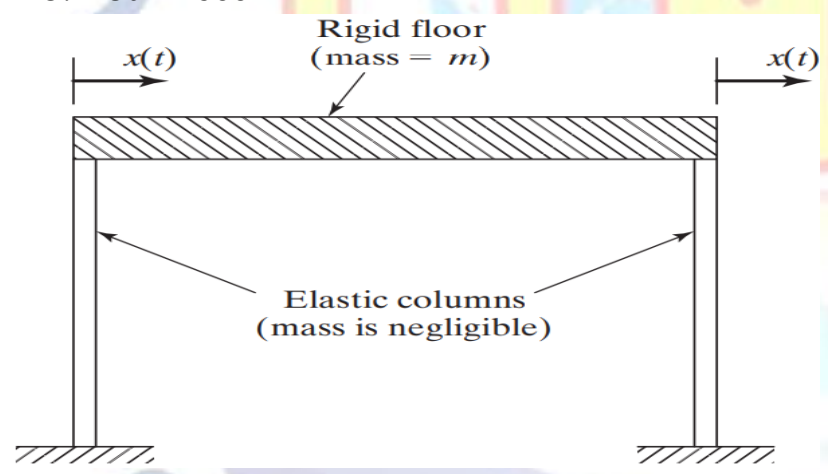

Fig. Building frame

It consists of both ends are fixed at bottom and elastic columns are supports rigid floor or mass on top.

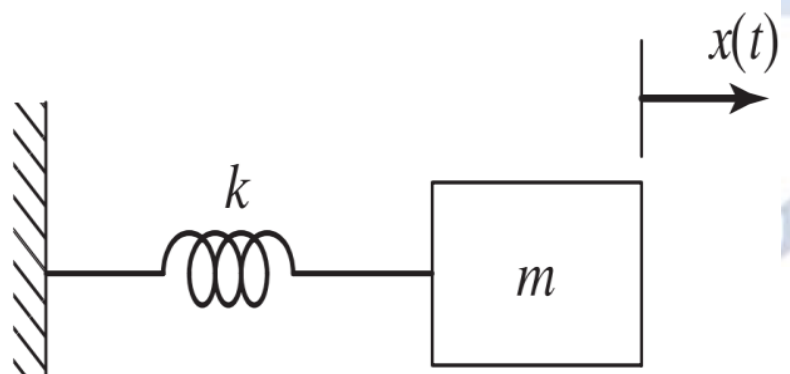

It consists of fixed support at one end and mass at bottom and it is subjected to the loading and stiffness spring are provided to connecting to a fixed end to mass

In this methodology, it is important to know about vibration and types of vibrations. To understanding about dynamic analysis of the structures, it is necessary to understand.

\section{Vibration}

Vibration is a motion of a system or body. The bodies have been displaced from the position of equilibrium and appearing as an oscillation.

\section{Types of Vibrations:}

\section{Free Vibration}

1. Free vibration of the system are defined as there is no external forces acting in the system. It is called as the free vibration system.

2. So no forces acting means no loss of energy in this type of system.

3. Example is oscillation of the simple pendulum.
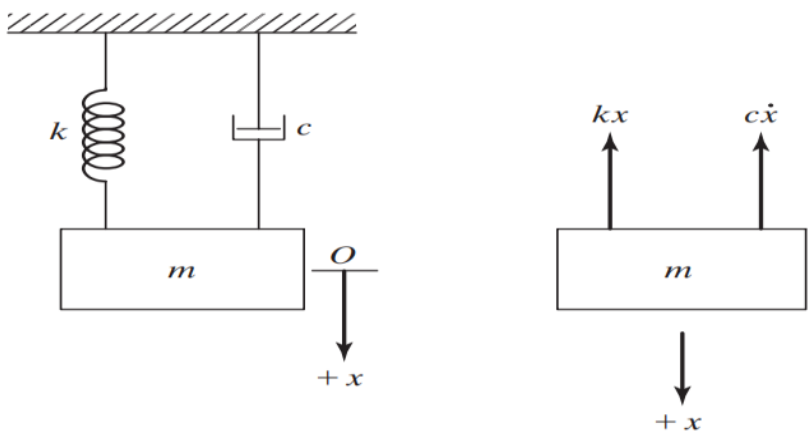

Fig. Free Vibration system

\section{Forced Vibration}

1. Forced vibration of the system are defined as there is external forces are acting on the system. It is known as the forced vibration system.

2. So in this forces are acting, which means here the loss of energy present in this system.

3. In this vibration, the system is forced to vibrate.

4. Example is spring mass system

Fig. the Spring Mass system 

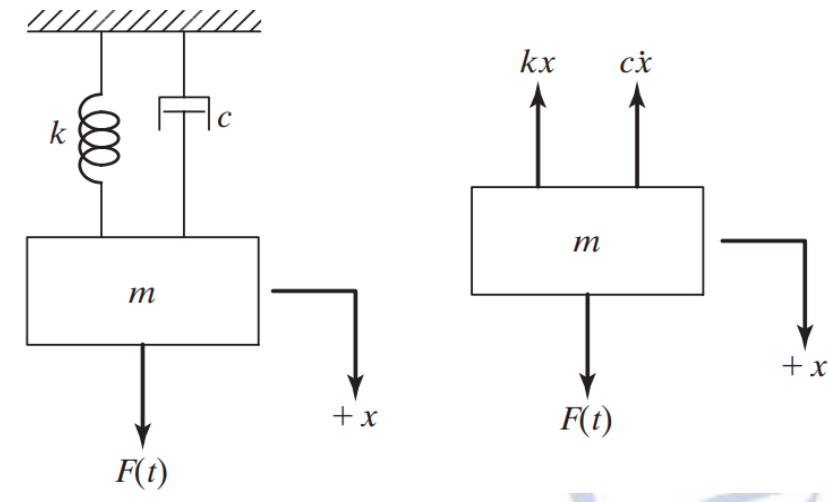

Fig. Forced Vibration

\section{Damped Vibration}

Damping vibration is the vibration, in this damping elements are attached to the system.

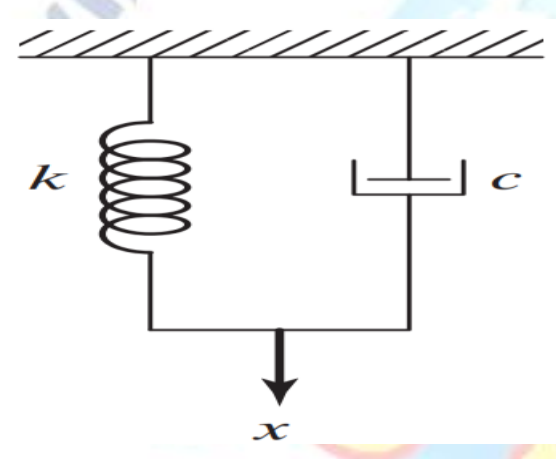

Fig. Damping Vibration

\section{Undamped Vibration}

Undamping vibration is the vibration, in this no damping elements are attached to the system.

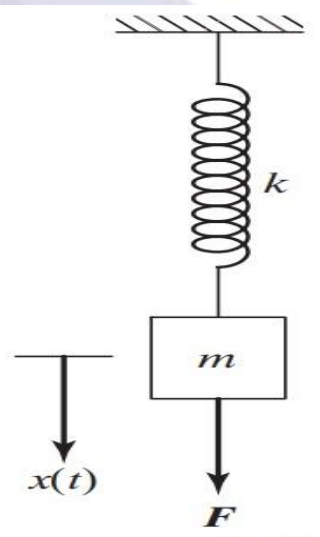

Fig. Undamping Vibration

The safety of the dynamic structures is important to understand about vibration and damping and degree of freedom of the structures. There are different types of principles are used for dynamic structures. Some of the important are as follows;

1. D'Alemberts principle
2. Newtons $2^{\text {nd }}$ law of motion

3. Hamiltons principle

4. Virtual work principle

Equation of motion for the general vibration of the system ;

\section{$m \ddot{x}+c \dot{x}+k x=f(t)$}

Degree of Freedom is important for the structures. It is the number of independent co ordinates to the structural system. It indicate the position of mass of the system at any instant time is known as the degree of freedom.

\section{Types of D.O.F. systems}

1. S.D.O.F. systems: if one co ordinate is sufficient to define the position or geometry of mass system at any time is called S.D.O.F. system.

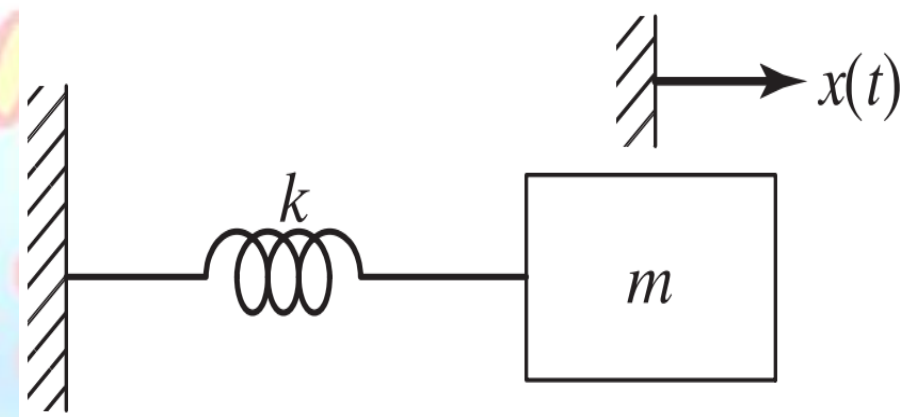

Fig. S.D.O.F. system

2. M.D.O.F. systems: if morethan one independent co ordinate is define the position or geometry of the mass system at any time is called as multi degree of freedom.

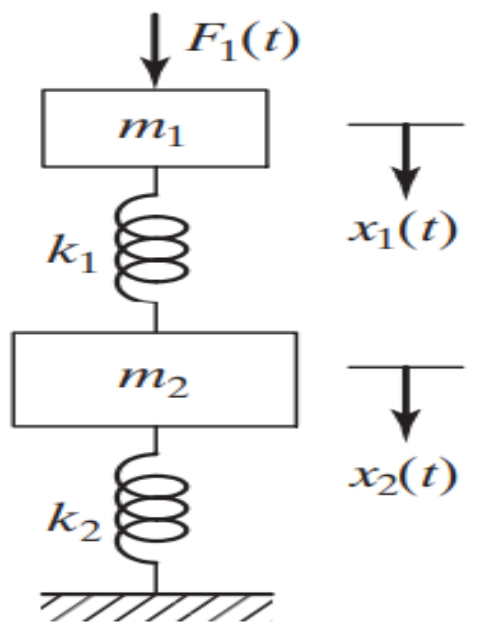

Fig. Multi degree of freedom system 
3. Continuous systems: If the mass of system to infinite degree of freedom is known as the continuous system.

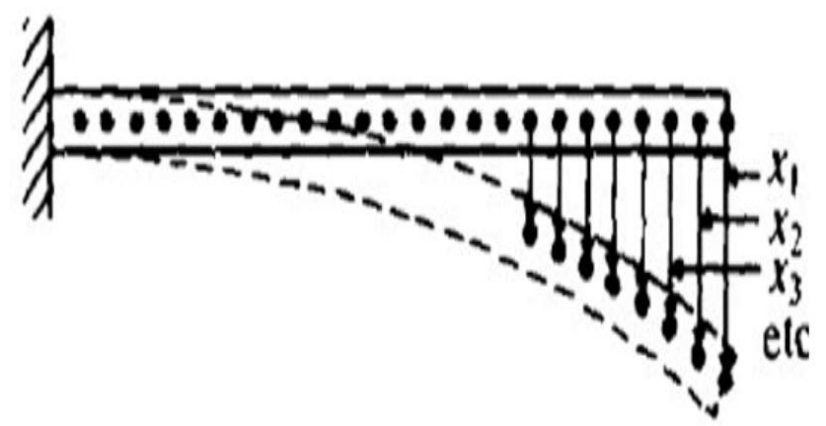

Fig. the Continuous system of cantilever beam

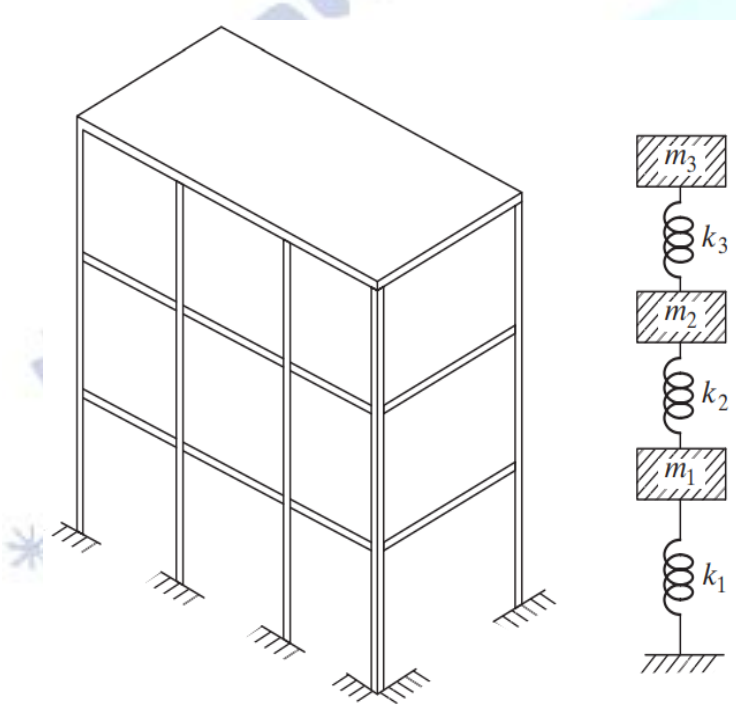

Fig. Storey building with details of the system

$$
\begin{aligned}
& \mathrm{c}=\text { damping } \\
& \mathrm{v}=\text { velocity } \\
& \mathrm{m}=\text { mass } \\
& \mathrm{g}=\text { acceleration due to the gravity }
\end{aligned}
$$

\section{ACKNOWLEDGEMENT}

My sincere thanks to Dr. A.R. Prakash and Dr. Dinesh Kumar; Mr. B. Dhavaleshwar Rao; Mr. Viqar Malik and Mr. Syed Riyaz and Dr. Bharathi Murugan ; Head of the department civil and structural engineering.

\section{v. CONCLUSION}

In this paper, The overall concept of structural dynamics is clearly explained. It is also necessary to understanding about special structures. So it place an important role in design aspects in structural engineering. In this paper also explains easy understanding and the introduction to dynamic structures. It is necessary to design of high rise structures. This study is also helpful to design and understanding of seismic structures.

\section{REFERENCES}

[1] Mechanical vibrations by Singiresu S. Rao

[2] Dynamics of structures by Anil K Chopra

[3] S.R.Damodarasamy; Basics of structural dynamics and seismic design

[4] Structural Dynamics by Mario paz Overview of structural Dynamics of structures 2019 The University of Qom By A. Shahiditabar

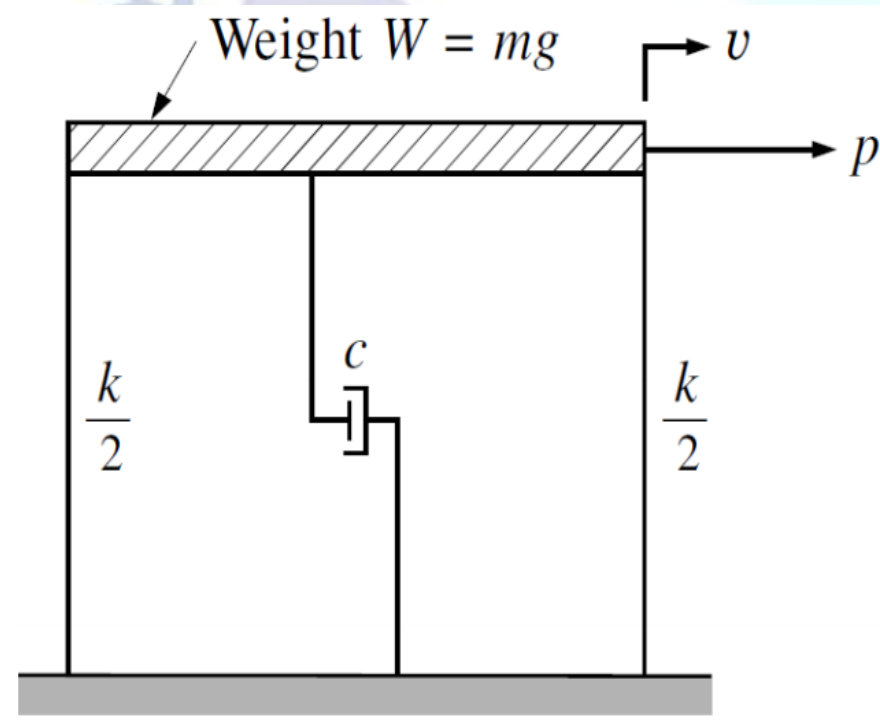

Fig. The Structural Building system

Where,

$\mathrm{p}=$ applied force
$\mathrm{k}=$ stiffness 